'Servicio de Radiología. Hospital San Juan de Dios de La Serena. La Serena, Chile. ${ }^{2}$ Servicio de Radiología. Clínica Elqui. La Serena, Chile.

${ }^{3}$ Programa de especialidad en Radiología. Oficina de Postgrado. Facultad de Medicina, Universidad Católica del Norte. Coquimbo, Chile. ${ }^{4}$ Departamento de Radiología, Pontificia Universidad Católica de Chile. Santiago, Chile. ${ }^{5}$ Sección de Análisis de Recursos de la Información. Unidad de Biblioteca, Universidad Católica del Norte. Coquimbo, Chile. aResidente de tercer año de Radiología Universidad Católica del Norte. Coquimbo, Chile.

bBibliotecóloga, Master en Documentación Digital.

Conflictos de interés y apoyo financiero: No hubo apoyo financiero para la realización de este trabajo.

Los autores declaran no tener conflictos de interés relacionados al contenido de este artículo, en especial, de no existir relaciones con las distintas entidades gratuitas o de acceso pagado referidas en este texto.

Recibido el 24 de febrero de 2017, aceptado el 26 de diciembre de 2017

Correspondencia a: Joaquín Hevia Morel

Av. El Santo 1475

Servicio de Radiología, Clínica Elqui. La Serena, IV región, Chile. drjoaquinhevia@gmail.com

\section{Herramientas útiles y métodos de búsqueda bibliográfica en PubMed: guía paso a paso para médicos}

\author{
JOAQUÍN HEVIA M. ${ }^{1,2,3}$, ÁLVARO HUETE G. ${ }^{4}$, \\ SANDRA ALFARO F. ${ }^{1, \mathrm{a}}$, VERÓNICA PALOMINOS V. ${ }^{5, \mathrm{~b}}$
}

\section{Useful tools and methods for literature retrieval in pubmed: step-by-step guide for physicians}

Developing skills to search the medical literature has potential benefits on patient care and allow physicians to better orient their efforts when answering daily clinical questions. The objective of this paper is to share useful tools for optimizing medical literature retrieval in MEDLINE using PubMed including MeSH terms, filters and connectors.

(Rev Med Chile 2017; 145: 1610-1618)

Key words: Information Storage and Retrieval; Internet; Medical Subject Headings; PubMed; Radiology.
$\mathrm{R}$ ealizar una adecuada búsqueda bibliográfica de literatura científica en la práctica clínica diaria implica un beneficio directo en el manejo del paciente, permitiendo actualización de los conocimientos médicos, implementación de conductas basadas en la evidencia científica y resolución con fundamentos de problemas clínicos cotidianos. Además de lo anterior, una búsqueda adecuada permite encontrar las referencias óptimas antes y durante la realización de una investigación científica ${ }^{1-8}$.

La información científica se duplica cada 5 años $^{9,10}$, siendo las revistas científicas el medio principal para la comunicación médica. Se puede acceder a ellas a través de internet, contando con variados repositorios en línea donde se pueden realizar búsquedas. Estos repositorios organizan la información recopilada de tal forma que se permita su fácil acceso, siendo MEDLINE la base de datos biomédica más utilizada, con mayor cantidad de contenido y de actualización diaria ${ }^{1}$.
Se puede acceder a ella gratuitamente a través del sitio PubMed: http://www.pubmed.gov, que es el motor de búsqueda Web-Based. La base de datos bibliográfica es recolectada por la National Library of Medicine USA. Ésta sólo incorpora detalles bibliográficos de los artículos de revistas y no la totalidad del contenido del artículo, por lo que entrega enlaces para acceder a los artículos completos. PubMed indexa más de 5.600 revistas de medicina y ciencias de la salud, de más de 70 países $^{1-5,7,9,10}$.

El poder desarrollar la habilidad de realizar búsquedas adecuadas en PubMed permite encontrar los artículos indicados para responder las interrogantes del quehacer médico en el menor tiempo posible y sin distracciones hacia artículos no requeridos. Si bien existen distintos productos comerciales que sintetizan grandes cantidades de información en revisiones de extensión reducida (como UpToDate ${ }^{\circledR}$ o StatDx ${ }^{\circledR}$ ), estos no siempre abarcan todas las dudas específicas de la práctica 
diaria ni contienen información tan actualizada como las bases de datos bibliográficas ${ }^{1,3,4,10,11}$.

El objetivo de este artículo es introducir a los médicos menos familiarizados con las búsquedas bibliográficas a esta poderosa herramienta: PubMed. Se utilizarán ejemplos que ayudarán a la comprensión y mejor aplicación de los conceptos relevantes. Es recomendable aplicar en forma sincrónica los pasos expuestos en un computador con acceso a internet, favoreciendo así su uso y aprendizaje inmediato.

Se debe tomar en consideración que la mayor parte del contenido de los repositorios se encuentra en inglés y que, al realizar búsquedas en ese idioma, se accederá a toda la literatura anglosajona y a la mayoría de la literatura indexada, aunque esté en otro idioma, ya que habitualmente las revistas científicas exigen incluir un resumen en inglés para su publicación ${ }^{9}$. Considerando lo anterior, si se busca en inglés, se encontrará en inglés y otros idiomas; mientras que al hacer búsquedas en español, los resultados se limitarán a ese idioma en particular. Por este motivo, los ejemplos usados en este artículo se encuentran en inglés, tal como se haría una búsqueda bibliográfica durante la actividad clínica del día a día.

\section{Herramientas y metodologías de búsqueda}

A continuación se enumeran algunas recomendaciones para optimizar las búsquedas en PubMed:

\section{1) Antes de la búsqueda $a^{4,8-10}$}

Se debe tener muy claro cuál es la pregunta clínica que se está haciendo y para la que se desea una respuesta. El factor que será más determinante en obtener resultados apropiados será disponer de una pregunta que cumpla con el mayor número posible de los siguientes criterios:

- Términos precisos (ver más adelante: MeSH terms). Un método para utilizar palabras adecuadas en una búsqueda es revisar algún artículo del tema que se está analizando. Normalmente esto orientará a considerar términos que no estaban siendo incluidos originalmente $^{4}$.

- Problema (duda clínica).

- Técnica de estudio/intervención (por ejemplo: alguna modalidad de imagen, algún fármaco utilizado, cierta técnica quirúrgica, etc.).
- Tipo de estudio o intervención (por ejemplo: en el caso de estudios de tomografía computada definir si son estudios con contraste iodado endovenoso, sin contraste endovenoso, con equipos muticanal, usando dosis reducida, etc.).

- Paciente (grupo etario, género, raza).

- Comparación (fármaco versus placebo, rayos $\mathrm{X}$ versus ultrasonido, etc).

\section{2) Errores habituales ${ }^{2,10}$}

Una búsqueda que arroje demasiados resultados o bibliografía que se aleje del tema en cuestión (irrelevante), hará que disminuya el interés del usuario por hacer nuevas búsquedas. Para ello se debe evitar:

a) Utilizar frases cortas: encontraremos demasiados resultados irrelevantes. Por ejemplo, si buscamos "HRCT in bronchiectasis" (inglés para "Bronquiectasias en tomografía computada (TC) de tórax de alta resolución") los resultados estarán formados por toda la literatura que incluya ambas palabras en cualquier parte del texto, independiente de si esos artículos están o no relacionados al tema de interés, como por ejemplo el primer artículo de la lista: "Impulse oscillometry in adults with bronchiectasis" (inglés para "Oscilometría de impulso en adultos con bronquiectasias").

b) Buscar sólo artículos gratuitos: podríamos perder información fundamental. Se debe recordar que muchas universidades chilenas e instituciones de salud tienen acceso a bibliotecas on-line con acceso a revistas médicas.

\section{3) Generación de una pregunta apropiada}

A modo de ejemplo, utilizaremos como pregunta clínica cuáles son los criterios diagnósticos para apendicitis aguda por tomografía computada en adultos:

"computed tomography diagnostic criteria appendicitis acute adults"

Se debe destacar que no influirá en el resultado el orden en que están escritos los distintos términos, que de forma predeterminada son unidos por AND (inglés para "Y") al escribir un espacio entre ellos. Esto será descrito en mayor detalle más adelante.

Al realizar esa búsqueda, PubMed arroja 67 resultados (las búsquedas del artículo fueron realizadas entre los días 11 y 13 de diciembre de 2016), a diferencia de los 14.254 resultados que el 
sistema arroja si se busca como "acute appendicitis diagnosis" (inglés para "apendicitis aguda diagnóstico"). Con este ejemplo se desea enfatizar que mientras más especificidad tenga la búsqueda, se obtendrá un menor número de resultados (que es el objetivo primario de utilizar PubMed: encontrar bibliografía de calidad, relevante para resolver una duda clínica determinada).

\section{4) Boolean operators (conectores) $)^{1,2,4,5,10}$}

Las búsquedas pueden ser refinadas utilizando los conectores: AND, OR y NOT (inglés para "Y", "O" y "NO"). Deben ser ingresados en mayúsculas.

AND: los resultados de la búsqueda deben tener todos los términos empleados.

OR: los resultados de la búsqueda pueden tener uno o más de los términos utilizados.

NOT: Se utiliza para eliminar un término del resultado de la búsqueda.

Por ejemplo, si se desea realizar la búsqueda del ejercicio descrito en el punto 3 sin incluir los estudios que utilicen ultrasonido (US), tendría que modificarse de la siguiente manera: "computed tomography diagnostic criteria appendicitis acute adults NOT ultrasound", lo que disminuye a 5 los resultados. Si fuera de interés comparar los criterios para TC y resonancia magnética $(\mathrm{RM})$ podría hacerse la busqueda de la siguiente forma: " $M R I$ AND computed tomography diagnostic criteria acute appendicitis adults" (al utilizar espacios PubMed entiende que se está utilizando el conector AND, por lo que sería lo mismo que escribir " $M R I$ computed tomography...”). Si se desea buscar los criterios para cualquiera de las dos técnicas (TC o RM) la búsqueda sería: "MRI OR computed tomography AND diagnostic criteria acute appendicitis adults".

Los operadores son procesados por PubMed en forma secuencial de izquierda a derecha ${ }^{2}$, por lo tanto el resultado será distinto en "....adults NOT ultrasound" y en "...ultrasound NOT adults".

\section{5) Registros de PubMed y MeSH terms s,2,4,5,8-10 $^{\text {(n) }}$}

Cada registro de PubMed consiste en campos requeridos por MEDLINE: Título, Autores, Afiliación, Resumen, Lenguaje, Fecha de publicación, Título de la Revista y MeSH (Medical Subject Heading, ingles para "Titulo de Tema Médico"), cada uno de los cuales se registra por separado y podrá ser utilizado como método para filtrar resultados de una búsqueda. El campo MeSH contiene términos elegidos dentro de 22.568 definiciones (específicas y estandarizadas). El vocabulario de términos $\mathrm{MeSH}$ es actualizado en forma continua y a cada artículo se le asignan los términos $\mathrm{MeSH}$ que mejor reflejan su contenido, según análisis de indexadores expertos. De esta forma se indexan los artículos basados en su contenido y no en las palabras que incluyen. En términos sencillos, utilizar un MeSH term (inglés para "término") permite incluir en los resultados todos los sinónimos, abreviaciones y traducciones de un término en particular.

Considerando lo anterior, para que en el ejemplo previamente utilizado se incluyan en los resultados todos los artículos relacionados a tomografía computada y no se excluyan aquellos que se refieran a abreviaciones como CT o TC, que son conceptos que de otra forma podrían ser eliminados por nuestro motor de búsqueda, sería de utilidad buscar con los MeSH terms. Para ello, se debe realizar una búsqueda de cada concepto por separado y agregarlos al motor de búsqueda, según se describe en la Figura 1.

Para aquellos usuarios cuyo manejo del idioma inglés sea limitado, la herramienta MeSH terms de PubMed es también útil, ya que ofrece la terminología más adecuada en forma automática. Por ejemplo, al buscar "head pain" (traducción literal al inglés de "dolor de cabeza"), se ofrecerá como primer resultado "headache" (inglés para "cefalea").

\section{6) Límites ${ }^{1,2,4,8,10}$}

PubMed permite refinar la búsqueda al seleccionar límites (Figura 2), que incluyen tipo de publicación, fecha de publicación, idioma, entre otros.

\section{7) Crear una cuenta personalizada en PubMed y resaltar palabras de interés en resultados de búsqueda}

Es un proceso gratuito y muy breve, que se completa siguiendo los siguientes pasos:

a) Ir a www.pubmed.gov y hacer click en "Sign in to NCBI" ubicado en la esquina superior derecha de la pantalla. Posteriormente hacer click en "Register for an account" y seguir las instrucciones.

b) Una vez registrado, hacer click en " $M y N C B I$ " (margen superior derecho de la pantalla) y a continuación en "NCBI site preferences" (ubicado justo debajo de "My NCBI"). 
Métodos de búsqueda bibliográfica en Pubmed para médicos - J. Hevia et al

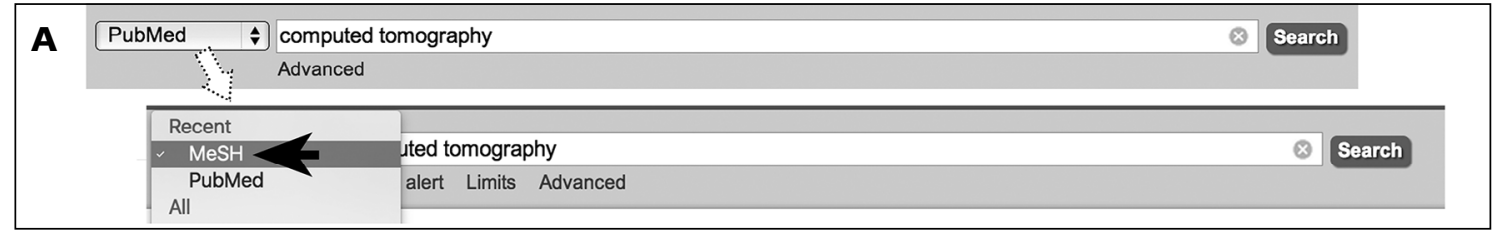

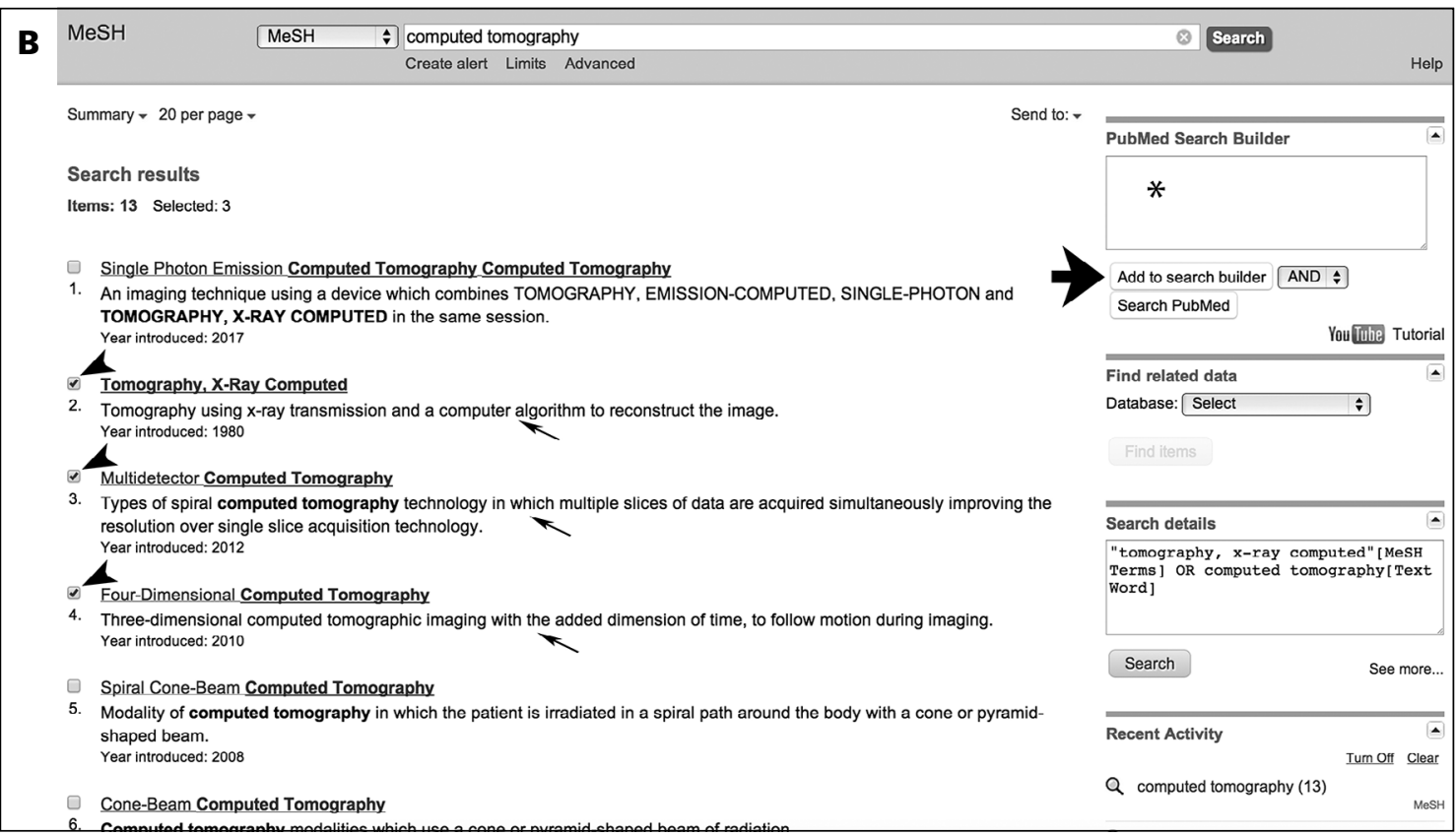

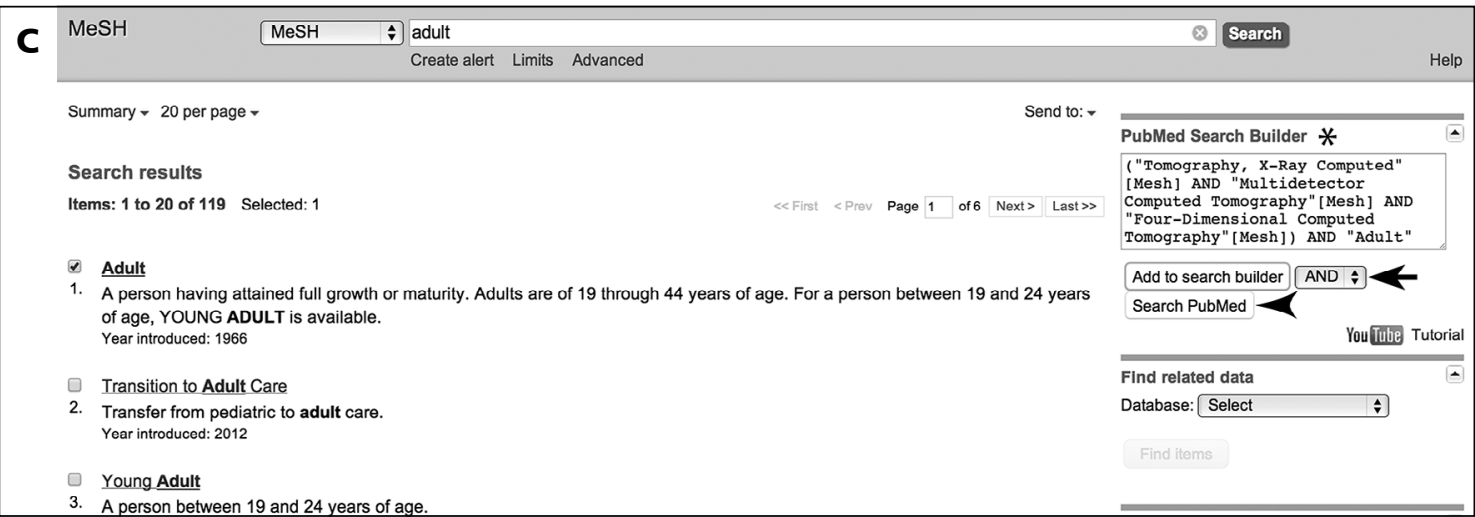

Figura 1. MeSH, sitio de selección de búsqueda de términos MeSH. A) Caja de búsqueda inicial. Hacer click en la caja expansible para seleccionar términos MeSH (Flecha rellena). Una vez seleccionado MeSH, realizar búsqueda. B) Resultados de la búsqueda MeSH. Se deben seleccionar los términos que se quiera incluir en la búsqueda (puntas de flecha), revisando que las definiciones correspondientes sean las que se necesiten (flechas delgadas). Para agregar las selecciones a la búsqueda, se debe presionar el botón "add to search builder" (flecha gruesa). Al presionar, se agregarán los MeSH terms correspondientes dentro de la caja de búsqueda (asterisco), como se muestra en C). C) Se puede seguir agregando MeSH terms repitiendo los pasos previos, recordando seleccionar el conector apropiado (AND, OR o NOT), según corresponda (Flecha) y al finalizar, hacer click en el botón "Search Pubmed" (punta de flecha). 


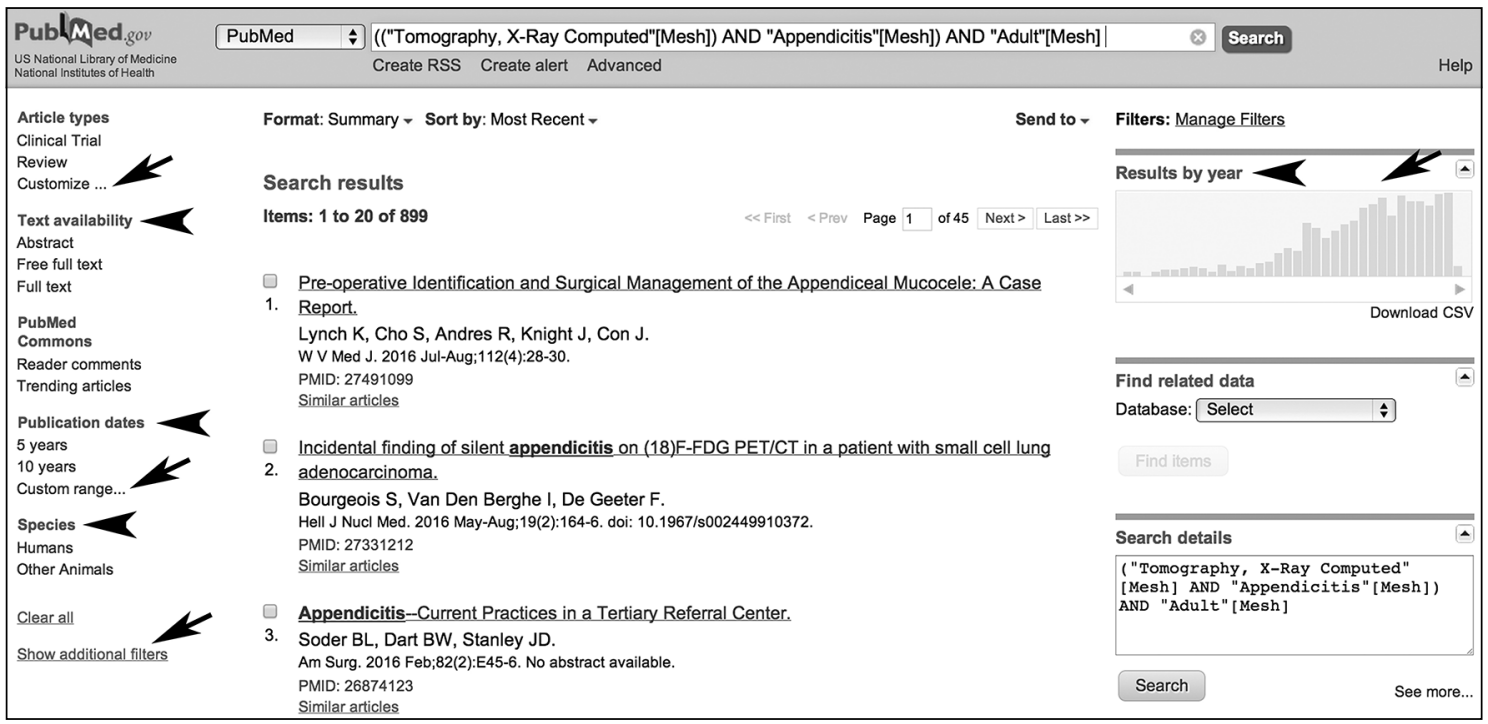

Figura 2. Límites. Se puede limitar los resultados según criterios (puntas de flecha) habituales o personalizados (flechas).

Tabla 1. Campos para búsquedas*

\begin{tabular}{|c|c|c|}
\hline \multicolumn{3}{|c|}{ Búsqueda utilizando campos y etiquetas (Tags) } \\
\hline Nombre del campo & Abreviatura & Descripción \\
\hline Título & {$[\mathrm{TI}]$} & La búsqueda se realizará solo en el título del artículo \\
\hline Título y Resumen & {$[T \mid A B]$} & Incluye resultados del título y el resumen (abstract) del artículo \\
\hline Autor & {$[\mathrm{AU}]$} & Búsqueda por nombre del autor \\
\hline $\begin{array}{l}\text { Encabezado de temas médicos } \\
\text { (MeSH terms) }\end{array}$ & [MESH] & $\begin{array}{l}\text { Búsqueda dentro de los términos biomédicos controlados (MeSH } \\
\text { terms) }\end{array}$ \\
\hline Lenguaje & {$[\mathrm{LA}] \circ[\mathrm{LANG}]$} & Idioma original del artículo \\
\hline Nombre de la revista & {$[T A] \circ[J O U R]$} & Abreviatura del título de la revista, título completo o número ISSN \\
\hline Fecha de publicación & {$[D P] \circ[P D A T]$} & Fecha en que el artículo fue publicado (en formato año-mes-día) \\
\hline
\end{tabular}

*Cada campo de referencia se identifica mediante una etiqueta que indica el campo específico en que se buscará el término. Para emplearlos se escribe el término de búsqueda seguido de la abreviatura del campo entre paréntesis cuadrados (etiqueta), lo que permite realizar la búsqueda dentro de ese único campo

c) En el subtítulo de "common preferences" (inglés para "preferencias frecuentes") deberá hacer click en "highlighting" (inglés para "destacar").

d) Elegir un color y posteriormente guardar los cambios en "save".

e) Ahora cada vez que se acceda a PubMed, puede ingresar como usuario registrado, lo que permitirá tener un historial de búsqueda individualizado, resaltar resultados y crear filtros de revistas de interés o por especialidad, como se detallará más adelante.

\section{8) Búsqueda por campos ${ }^{2}$}

Cada registro de PubMed contiene campos (ver punto 5). El usuario puede explicitar en qué campo desea buscar qué concepto (Tabla 1). Por ejemplo:

a) Si se desea buscar "hipertensión pulmonar y enfermedad pulmonar intersticial en tomografía computada" y que en el resultado sólo aparezcan artículos con esas palabras en el título, se debe buscar "pulmonary hypertension [ti] interstitial lung disease [ti] CT[ti]" 
b) Si se desea agregar al autor, se debe añadir "Wells[au]". Esto es especialmente útil cuando el apellido del autor es también una enfermedad o signo clínico (por ejemplo: Wilson).

c) Una de las fórmulas más efectivas es la búsqueda de los términos adecuados tanto en el título como en el resumen, lo que se realiza añadiendo "[tiab]". Siguiendo el ejemplo anterior, quedaría así: "pulmonary hypertension[tiab] interstitial lung disease[tiab] CT[tiab]". De esta forma se eliminarán resultados inútiles, donde esas palabras pudieran haber sido nombradas en el resto del texto, aumentando así la especificidad de nuestra búsqueda, sin perder tantos artículos como ocurrirá al buscar sólo en el campo "títulos". Un problema posible es que se agregarán resultados en que las palabras pueden estar pero los artículos no serán de ese tema en particular: En el ejemplo veremos como en los resultados aparecerán artículos como "hipertensión pulmonar en polimiositis" debido a que se mencionan también los conceptos "tomografía computada" y "enfermedad pulmonar intersticial” dentro del abstract.

\section{9) Alertas $^{2}$}

Si se desea profundizar en un tema particular y realizar una revisión bibliográfica o bien preparar un seminario de actualización es posible mantenerse al día incluso después de realizada la búsqueda índice, al pedirle a PubMed que envíe una alerta al correo electrónico cuando se agregue algún artículo nuevo a PubMed que cumpla con los criterios de nuestra búsqueda. Para hacerlo se deben seguir los pasos de la Figura 3.

\section{0) Clipboard (portapapeles) y Collections ${\text { (colecciones })^{2}}^{2}$}

El portapapeles permite dejar guardadas algunas referencias de los resultados de una búsqueda para ser revisadas más tarde (Figura 4). Los elementos del portapapeles permanecen guardados durante $8 \mathrm{~h}$. Si se desean guardar por tiempo ilimitado se debe hacer dentro de "Collections", siguiendo los pasos de la misma figura. Para rescatar referencias guardadas en colecciones, ingrese a "My NCBI".

\begin{tabular}{|c|c|}
\hline PubMed & pulmonary hypertension[tiab] interstitial lung disease[tiab] CT[tiab] \\
Create RSS Create alert Advanced & Search \\
\hline Format: Summary - Sort by: Most Recent & Send to - Filter your results: \\
\hline
\end{tabular}

Figura 3. Recibir alertas cuando nuevos artículos relacionados a una búsqueda se agreguen a PubMed. Hacer click en "create alert" (flecha) y seguir los pasos de la ventana emergente que se abrirá en el explorador, seleccionando la frecuencia con que se quiera recibir correos de alerta.

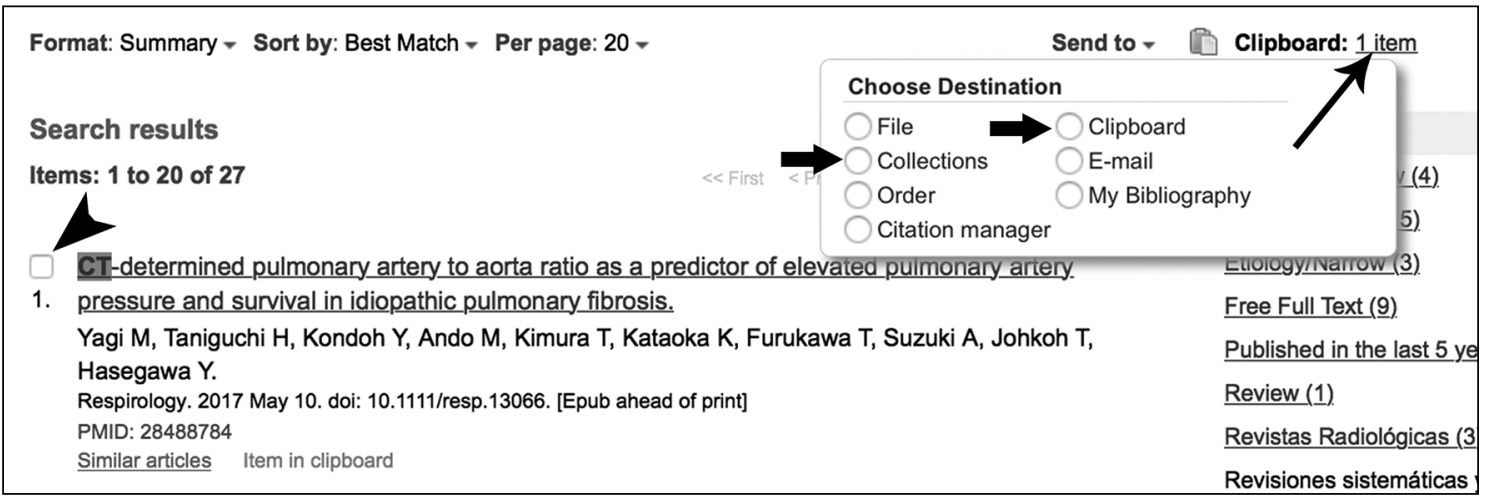

Figura 4. Guardar referencias en Clipboard (portapapeles) o en Collections (colecciones), para ser revisadas más tarde. Se debe seleccionar las referencias que quieran ser guardadas haciendo click en las cajas (punta de flecha) y posteriormente seleccionar la carpeta donde se desee guardar (flecha gruesa). Una vez guardadas las referencias, se puede revisar el portapapeles haciendo click en "Clipboard" (flecha delgada). 


\section{1) Filtro de revistas de interés ${ }^{2,5}$}

En la experiencia de los autores, una de las formas más útiles de acotar los resultados a artículos de interés para interrogantes del día a día es crear un filtro personalizado que nos permita obtener los resultados de artículos publicados en revistas de la especialidad (Figura 5).

Para crear un filtro personalizado (de revistas radiológicas en el caso de los autores o cualquier otra especialidad) se debe ingresar con nuestro usuario de PubMed. Posteriormente hacer click en "My NCBI" (tal como se expuso en el paso número 7) y en el apartado de filtros ("filters"), hacer click en "manage filters" (inglés para "administrar filtros"). Se abrirá una nueva ventana: hacer click en "create custom filter" (inglés para "crear filtro personalizado"). En el cuadro "query terms" (inglés para "términos de búsqueda") debemos escribir todo lo que deseamos agregar al filtro. Resulta útil agregar aquellas revistas de nuestro interés o términos que aparezcan en el general de las revistas de la especialidad, siempre seguidas de "[jour]" y conectadas por "OR" (Figura 6). A modo de ejemplo, en el caso de revistas del ámbito de la radiología, una fórmula últil es utilizar términos comunes como: Radiol[jour] OR Roentgenol[jour] OR Imaging[jour]. Con estos se incluirán en el filtro la mayoría de las revistas más reconocidas, sin embargo, algunas de nuestro interés podrían quedar fuera (por ejemplo: $R a$ diographics) si no cumplen con los criterios, por lo que la podemos agregar en forma explícita: ... OR Radiographics[jour].

Una vez agregados los términos deseados, es necesario elegir un nombre para nuestro filtro y guardarlo. Para poder utilizar el filtro creado en búsquedas futuras, primero debe ser activado (según se describe en la segunda parte de la Figura 6).

\section{2) Filtro de revisiones sistemáticas:}

Si se siguen nuevamente los pasos anteriores (ver apartado número 11), al crear un filtro escribiendo en los "query terms": "Cochrane Database Syst Rev[jour] OR systematic review [ti]", se puede obtener un instrumento de búsqueda que arroje como resultados sólo revisiones sistemáticas. Esto difiere de filtrar utilizando el límite "review" (inglés para "revisión") según lo descrito en el apartado de Límites (número 6), donde en los resultados se incluirán revisiones narrativas $o$ "artículos de revisión" que corresponden a revisiones descriptivas de literatura seleccionada por un experto respecto a un tema. $\mathrm{Al}$ crear un filtro personalizado en los "query terms" para revisiones sistemáticas sólo se obtendrán artículos que se ajusten a esta descripción, es decir, revisión y análisis de todos los artículos relevantes respecto a un tema, revisados por varios expertos, que con frecuencia incluyen métodos estadísticos para sintetizar los datos de múltiples estudios en una estimación cuantitativa ${ }^{12}$.

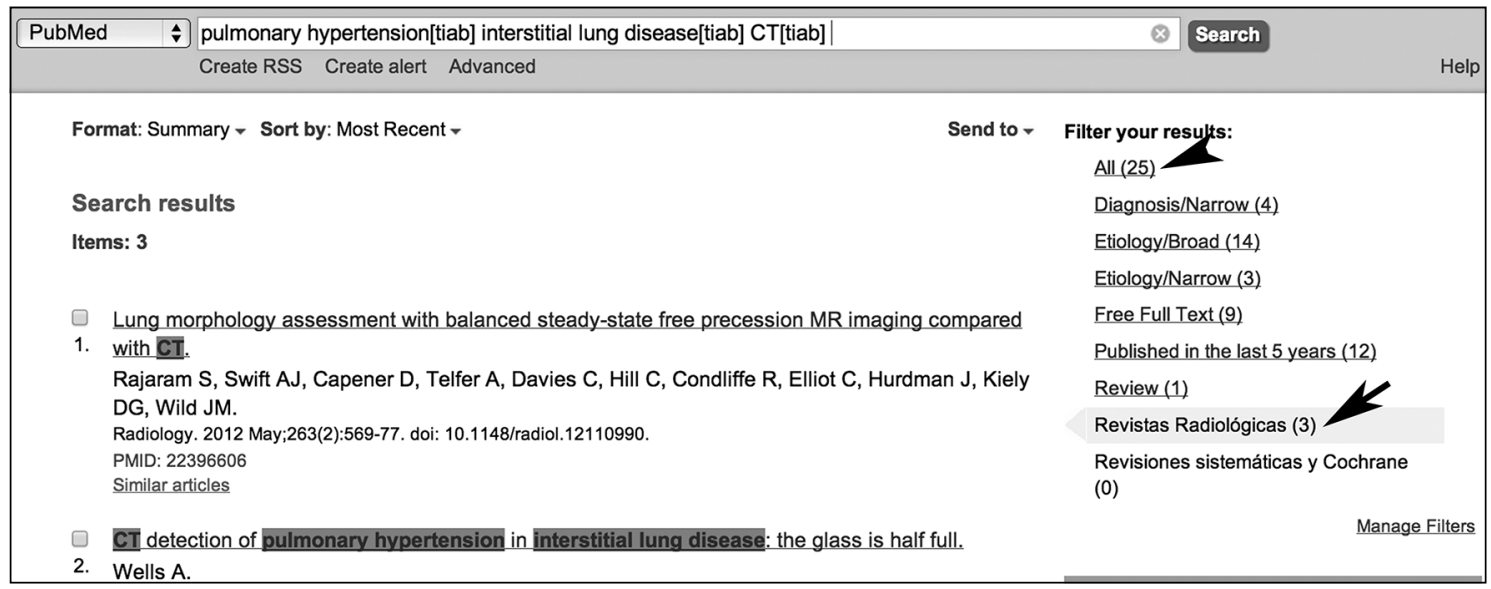

Figura 5. Disminuir número de artículos en resultados utilizando filtro de revistas radiológicas. Al utilizar el filtro en el caso del ejemplo, se disminuirá el número de resultados de 25 (punta de flecha) a 3 (flecha). Estos 3 artículos serán aquellos publicados en revistas radiológicas que cumplan con los criterios del filtro personalizado de los autores. 


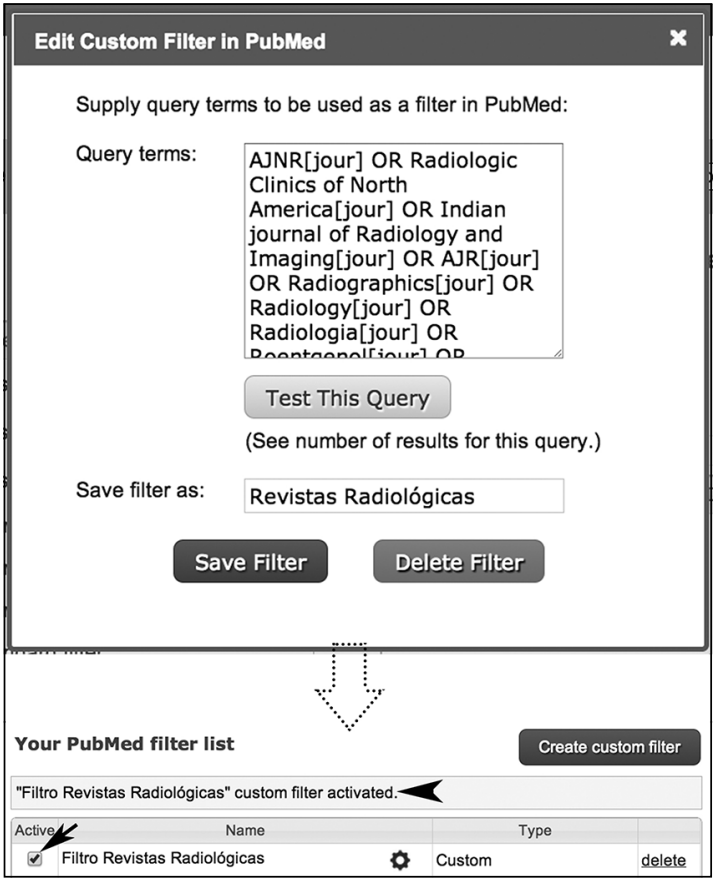

Figura 6. Ejemplo de "query terms" para crear un filtro de revistas radiológicas. Una vez escritos los términos de búsqueda para nuestro filtro, se debe guardar. Posteriormente se debe activar el filtro creado para poder ser utilizado en las futuras búsquedas. Para ello se debe hacer click en la casilla "active" (flecha rellena). Aparecerá el mensaje "Filter activated" (punta de flecha).

\section{3) $\mathrm{Geek}^{1,4,7}$}

Debemos hacer las búsquedas (en PubMed o Google) como lo haría un experto en informática. Existen dos herramientas muy útiles que son aplicables para nuestra práctica médica:

a) Utilizar el símbolo de asterisco $\left(^{\star}\right)$ cuando se desee buscar por el inicio de una palabra e incluir en los resultados todos los tipos de terminaciones asociadas: por ejemplo, si buscamos "ultras" incluiremos en los resultados palabras que se inicien con "ultras" como "ultrasound", "ultrasonography" y "ultrasonic", o si buscamos "appendi*" incluiremos palabras como "appendicitis", "appendix" y "appendiceal".

b) Al buscar términos compuestos por múltiples palabras, si los escribimos entre comillas (“), el buscador sólo incluirá resultados en que los términos se encuentren escritos en esa forma exacta, ya que considerará que es un

\begin{abstract}
"único elemento de búsqueda". Por ejemplo si buscamos $<M R I$ findings "Wilson disease" $>$ (ingles para $<$ Hallazgos de RM "Enfermedad de Wilson" $>$ ) eliminaremos resultados que tengan las palabras "Wilson" y "disease" por separado: 113 resultados útiles, a diferencia de 726 resultados (la mayoría inapropiados) si no incluimos los símbolos para convertir ambas palabras en un único elemento de búsqueda.
\end{abstract}

\section{Conclusión}

PubMed es una herramienta poderosa para realizar búsquedas de literatura médica. Al utilizar las herramientas y técnicas entregadas en este artículo, es de esperar que tanto médicos como otros profesionales de salud puedan ir perfeccionando y desarrollando sus habilidades para encontrar literatura científica de utilidad tanto para su práctica diaria como para hacer revisiones bibliográficas más profundas. Mientras más se practique la utilización de las herramientas descritas, el acto de realizar búsquedas bibliográficas será intuitivo, rápido y efectivo.

\section{Referencias}

1. Vincent B, Vincent M, Ferreira CG. The Community Oncologist Making PubMed Searching Simple: Learning to Retrieve Medical Literature Through Interactive Problem Solving. Oncol 2006; 11: 243-51.

2. Sriganesh V. Using PubMed in radiology: Ten useful tips for radiologists. Indian J Radiol Imaging 2011; 21 (3): 162-9.

3. Thakre SB, Thakre S SS, Thakre AD. Electronic biomedical literature search for budding researcher. J Clin Diagnostic Res 2013; 7 (9): 2033-7.

4. Moraga J, Manterola C, Ricardo C, Urrutia S. ¿Dónde Y Cómo Buscar Evidencia Científica En Medicina? Rev Chil Cir 2014; 66 (5): 502-7.

5. Muñoz Núñez CF, Sendra Portero F. [Google and PubMed for physicians: how to find information without getting lost]. Radiologia 2013; 55 (1): S37-46.

6. Sharpe RE, Sharpe M, Siegel E, Siddiqui K. Utilization of a radiology-centric search engine. J Digit Imaging 2010; 23 (2): 211-6.

7. Rau JL. Searching the literature and selecting the right references. Respir Care 2004; 49 (10): 1242-5. 
8. Rada G, Andrade M, Leyton L, Pacheco C, Ramos E. Búsqueda de información en medicina basada en evidencia. Rev Med Chile 2004; 132: 253-9.

9. Sood A, Ghosh AK. Literature Search Using PubMed: An Essential Tool for Practicing Evidence-Based Medicine. J Assoc Physicians India 2006; 54: 303-8.

10. Ebbert JO, Dupras DM, Erwin PJ. Searching the Medical
Literature Using PubMed: A Tutorial. Mayo Clin Proc 2003; 7878 (87): 87-91.

11. Islamaj Dogan R, Murray GC, Neveol A, Lu Z. Understanding PubMed user search behavior through log analysis. Database 2009; 2009: bap018.

12. Uman, LS. Systematic Reviews and Meta-Analyses. J Can Acad Child Adolesc Psychiatry 2011; 20 (1): 57-9. 